\title{
The Role of Pain Catastrophizing in the Prediction of Acute Procedure Related Pain
}

\author{
Liuda Brogiene ${ }^{1}$, Lolita Grygalyte ${ }^{* 2}$, Benas Kakta ${ }^{2}$ and Andrius Macas ${ }^{1}$ \\ ${ }^{1}$ Department of Anesthesiology, Lithuanian university of Health Sciences hospital Kaunas clinics, Kaunas, Lithuania \\ ${ }^{2}$ Faculty of Medicine, Academy of Medicine, Lithuanian university of Health Sciences, Kaunas, Lithuania
}

*Corresponding author: Lolita Grygalyte, Faculty of Medicine, Academy of Medicine, Lithuanian university of Health Sciences, A.

Mickeviciaus st., 9, LT-44307 Kaunas, Lithuania

ARTICLE INFO
Received: 慧April 28, 2021
Published: 业 May 06, 2021

Citation: Liuda Brogiene, Lolita Grygalyte, Benas Kakta, Andrius Macas. The Role of Pain Catastrophizing in the Prediction of Acute Procedure Related Pain. Biomed J Sci \& Tech Res 35(4)-2021. BJSTR. MS.ID.005726.

Keywords: Pain Catastrophizing; Acute Pain; Access-Site Pain; Percutaneous Coronary Intervention

Abbreviations: PCI: Percutaneous Coronary Intervention; CABG: Coronary Artery Bypass Grafting; PCS: Pain Catastrophizing Scale; SD = Standard Deviation; BMI: Body Mass Index; NRS: Numeric Rating Scale; CI: Confidence Interval; LB = Lower Bound; UB = Upper Bound; SE B = Standard Error of the Coefficient; OR: Odds Ratio

\section{ABSTRACT}

Background: Aim of this study was to assess preoperative pain catastrophizing scale (PCS) scores and its predictive value regarding acute access-site pain after percutaneous coronary intervention (PCI).

Methods: Patients who received PCI via radial artery enrolled in a prospective observational study. Patients filled out PCS questionnaire prior to procedure. Pain intensity was assessed using numeric rating scale during PCI and 2 hours, 12 hours, 24 hours, 48 hours, 1 week and 1 month after PCI.

Results: Median PCS score was 15 (8.0-22.0), female score (18.0 (10.0-29.5)) was higher than male $(14.0(6.5-20.0)(\mathrm{P}=0.030)$. Magnification scores were higher among females $\mathrm{P}=0.018)$. Patients aged 75 and older had highest PCS scores (20.0) and rumination scores $(\mathrm{P}=0.04$ and $\mathrm{P}=0.006$ respectively). Female pain scores during procedure $(2.5(0.0-5.0)$ and 2 hours after procedure $(4.0(2.0-5.0)$ were higher than male $(\mathrm{P}=0.024$ and $\mathrm{P}=0.013$ respectively). Significant correlation was found between gender and pain during PCI $(\mathrm{r}=0.219, \mathrm{P}=0.022), 2$ hours after $\mathrm{PCI}(\mathrm{r}=0.233, \mathrm{P}=0.015)$. We didn't find any correlation or predictive value of PCS for acute pain. Female sex predicted pain 1 month after PCI ( (OR = 3.143 95\% CI (1.063-9.296), P = 0.038).

Conclusion: PCS had no significant associations with pain after PCI. Females were more likely to report higher PCS and pain scores than males. Patients aged 75 and over reported higher PCS scores. Further research is needed to evaluate the importance of psychological factors regarding acute postprocedural pain.

\section{Introduction}

Myocardial revascularization by either percutaneous coronary intervention (PCI) or coronary artery bypass grafting (CABG) combined with pharmacotherapy remains the main approach in coronary artery disease treatment, PCI being a more preferred revascularization method in most cases [1-3]. Radial artery approach is generally favored over femoral artery approach, for the reason that transradial PCI has a lower incidence of access site complications, bleeding, lower risk of all-cause mortality and higher patient satisfaction compared to approach via femoral artery [4,5]. Despite numerous transradial approach benefits, patients undergoing PCI via radial artery experience pain during and after the procedure. There are several risk factors contributing to pain intensity during transradial access PCI, such as female sex, diabetes, smoking, low body mass index [6]. None of them have a direct connection to mental state and there are very limited data regarding the psychological risk factors for higher pain levels during and after PCI.

Nevertheless, various studies have shown the important role of depression, anxiety and pain catastrophizing as relevant independent risk factors of pain intensity experienced by patients in 
multiple surgical procedures $[7,8]$. Catastrophizing is described as a tendency to aggravate possible outcomes when subjected to painrelated stimuli. It is considered a result of dysfunctional cognitive and emotional adaptation mechanism and consists of three main components: rumination, helplessness and magnification [9]. Pain catastrophizing causes higher pain intensity, which in turn requires a more aggressive approach to postsurgical analgesia. Furthermore, it can lead to the development of chronic postsurgical pain, contribute to anxiety, depression or other negative emotional dysfunctions, overall, negatively affecting patient's quality of life. Pain catastrophizing scale (PCS) is widely used for objectifying rather abstract components of catastrophizing and is considered a standard measuring tool, as it is proven to be accurate, consistent and reliable $[8,10]$.

The primary aim of this study is to evaluate the relationship between pain catastrophizing and acute access site pain intensity after percutaneous coronary intervention via radial artery and determination whether pain catastrophizing scale score can be used as a predictive factor of acute pain after transradial PCI. Secondary aims include evaluating gender and age differences regarding PCS scores as well as pain scores up to 1 month after the procedure.

\section{Methods}

The Regional Ethics Committee approved the trial (approval number BE-2-7, 2018-02-26). 137 patients who underwent percutaneous coronary intervention via radial artery were enrolled in a prospective observational study (between 2018 and 2020).

\section{Inclusion Criteria}

a. Patients hospitalized in Lithuanian university of health sciences hospital Kaunas clinics Department of Cardiology for a percutaneous coronary intervention via radial artery (PCI).

b. Patients of both sexes and over the age of 18 .

c. Conscious patients, who gave written informed consent to participate in this study.

Due to missing data a total of 28 patients were excluded of whom 26 did not complete the PCS and 2 were unavailable for the follow-up pain assessment, leaving 109 patients for analysis (Figure 1). A prospective study with follow-up questions up to 1 month after the intervention was performed. Patients who were scheduled for PCI and agreed on participating in this study gave written informed consent.

The participants were aware that the information collected was confidential. Patient characteristic data including sex, age, BMI and comorbidities were collected. The patients were asked to fill out a questionnaire prior to the intervention on Pain Catastrophizing Scale (PCS). The PCS is consisted of thirteen remarks considering what the patient may experience when they are in pain. Patients have to rate their thoughts on each item from 0 ("not at all") to 4 ("all the time") (Table 1) [11]. The pain intensity was assessed by using the numeric rating scale (NRS), where $0=$ no pain and $10=$ worst pain imaginable 12. Patients were asked to rate their pain during the procedure and at 2 hours, 12 hours, 24 hours, 48 hours, 1 week and 1 month after the PCI. Statistical analysis was performed using IBMS SPSS 23.0. Visual inspections of Q-Q plots and Shapiro-Wilk goodness-of-fit tests were used to determine whether or not the data were normally distributed. Normally distributed continuous variables are presented as means with standard deviation and categorical data are presented as frequency and percentage.

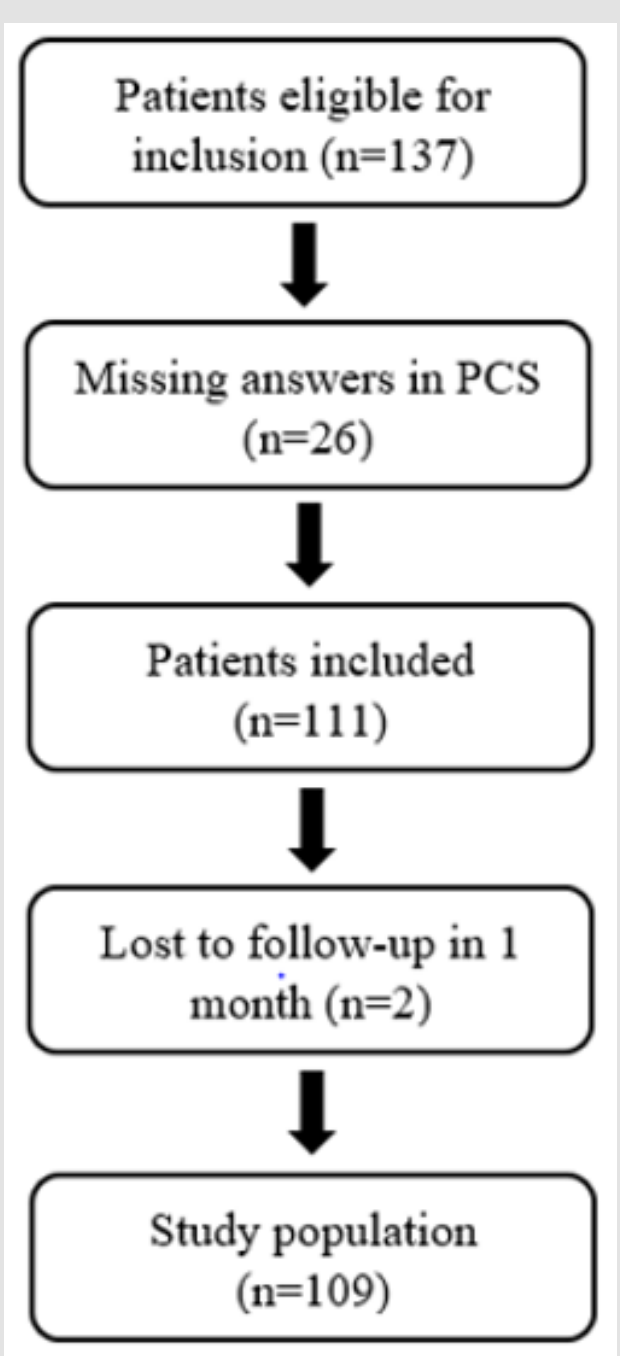

Figure 1: Flow chart.

The preoperative PCS scores were not normally distributed, as well as pain scores at all time points were not normally distributed and therefore results are presented as medians (with quartiles). A Mann-Whitney U test was run to determine if there were differences in overall PCS score and in pain scores between males and females. The Kruskal-Wallis $\mathrm{H}$ test was used to evaluate the distribution of PCS scores between age groups. Pearson correlations test was 
used to examine the associations between catastrophizing, pain and age and to study the associations between pain scores and age. Point-Biserial correlation test was used to examine the correlation between gender and pain scores. To investigate the association between preprocedural pain catastrophizing and postprocedural pain intensity and to determine whether PCS could be used as a predictive factor for pain intensity after the procedure, we used a linear regression test with postoperative pain intensity as the dependent variable. Univariate logistic regression analysis was carried out to examine PCS, sex and age as prognostic factors for acute pain. The results were considered statistically significant at $\mathrm{P}<0.05$.

Table 1: Pain Catastrophizing Scale.

\begin{tabular}{|c|}
\hline $\begin{array}{c}0 \text { - not at all, } 1 \text { - to a Slight Degree, } 2 \text { - to a Moderate Degree, } 3 \text { - to a } \\
\text { Great Degree, } 4 \text { - all the time }\end{array}$ \\
\hline When I'm in pain ... \\
\hline 1. I worry all the time about whether the pain will end. \\
\hline 2. I feel I can't go on. \\
\hline 3. It's terrible and I think it's never going to get any better. \\
\hline 4. It's awful and I feel that it overwhelms me. \\
\hline 5. I feel I can't stand it anymore. \\
\hline 6. I become afraid that the pain will get worse. \\
\hline 7. I keep thinking of other painful events. \\
\hline 8. I anxiously want the pain to go away. \\
\hline 9. I can't seem to keep it out of my mind. \\
\hline 10. I keep thinking about how much it hurts. \\
\hline 11. I keep thinking about how badly I want the pain to stop. \\
\hline 12. There's nothing I can do to reduce the intensity of the pain. \\
\hline 13. I wonder whether something serious may happen. \\
\hline Rumination: Sum of items 8, 9, 10, 11 \\
\hline Magnification: Sum of items 6, 7, 13 \\
\hline Helplessness: Sum of items 1, 2, 3, 4, 5, 12 \\
\hline
\end{tabular}

\section{Results}

In total 109 patients were included in the statistical analysis: 73 men (67\%) and 36 women (33\%). Baseline and demographic characteristics are presented in (Table 2). The overall median preoperative PCS score was 15.0 (8.0-22.0). The median for females was 18.0 (10.0-29.5) and for males 14.0 (6.5-20.0). Overall PCS scores for females were statistically significantly higher than for males $(P=0.030)$. In addition to that, females had a statistically significantly higher magnification score in PCS than males $(\mathrm{P}=$ 0.018). Distributions of PCS scores were similar for all age groups, as assessed by visual inspection of a boxplot. Median PCS scores were statistically significantly different between groups, with the highest median score (20.0) being in the $\geq 75$ years old group and the lowest (9.0) among patients younger than 55 years old $(\mathrm{P}=$ 0.04). Rumination score was also higher among patients older than 75 years $(\mathrm{P}=0.006)$. PCS values are presented in (Table 3$)$. The median pain score during the procedure was $1.0(0.0-4.0)$.

Table 2: Demographic characteristics of the study population (n $=109$ ).

\begin{tabular}{|c|c|}
\hline Characteristics & n $(\%)$ or mean \pm SD \\
\hline Age (years) & $64.29 \pm 11.2$ \\
\hline \multicolumn{2}{|c|}{ Age groups } \\
\hline$<55$ years & $17(15.6)$ \\
\hline $55-59$ years & $12(11)$ \\
\hline 60-64 years & $26(23.9)$ \\
\hline $65-69$ years & $20(18.3)$ \\
\hline 70-74 years & $13(11.9)$ \\
\hline$\geq 75$ years & $19(17.4)$ \\
\hline Female sex & $36(33)$ \\
\hline Male sex & $73(67)$ \\
\hline BMI $\left(\mathrm{kg} / \mathrm{m}^{2}\right)$ & $29.07 \pm 5.5$ \\
\hline $\begin{array}{l}\text { Ischemic heart disease duration } \\
\text { (years) }\end{array}$ & $4 \pm 5.3$ \\
\hline Current smoker & $36(33)$ \\
\hline Diabetes mellitus & $16(14.7)$ \\
\hline Arterial hypertension & $99(90.8)$ \\
\hline Depression & $2(1.8)$ \\
\hline Dyslipidemia & $68(62.4)$ \\
\hline Emergency procedure & $19(17.4)$ \\
\hline Duration of PCI (minutes) & $33.39 \pm 20.23$ \\
\hline Times PCI performed & $1.67 \pm 1.001$ \\
\hline
\end{tabular}

Table 3: Demographic characteristics of the study population $(n=109)$.

\begin{tabular}{|c|c|c|c|c|}
\hline Characteristic & PCS, Median & Rumination, Median & Magnification, Median & Helplessness, Median \\
\hline \multicolumn{5}{|c|}{ sex } \\
\hline Male & $14.0(6.5-20.0)$ & $4.0(1.0-8.0)$ & $3.0(1.0-5.0)$ & $5.0(2.0-9.0)$ \\
\hline \multirow[t]{2}{*}{ Female } & $18.0(10.0-29.5)$ & $6.0(2.5-10.5)$ & $5.0(2.0-8.0)$ & $7.0(4.0-11.0)$ \\
\hline & $\mathrm{P}=0.030^{*}$ & $P=0.056^{*}$ & $P=0.018^{*}$ & $\mathrm{P}=0.051^{*}$ \\
\hline \multicolumn{5}{|c|}{ Age group } \\
\hline$<55$ years & $9.0(7.0-22.0)$ & $3.0(1.0-5.0)$ & $2.0(1.0-4.5)$ & $5.0(3.0-9.5)$ \\
\hline $55-59$ years & $17.0(8.0-20.0)$ & $5.0(2.0-8.0)$ & $3.0(1.5-5.5)$ & $6.0(2.5-9.0)$ \\
\hline
\end{tabular}




\begin{tabular}{|c|c|c|c|c|}
\hline 60-64 years & $14.5(8.0-22.0)$ & $5.0(2.0-10.0)$ & $4.0(2.0-7.0)$ & $7.0(2.0-10.0)$ \\
\hline 65-69 years & $10.0(5.0-18.0)$ & $2.0(1.0-5.0)$ & $2.0(1.0-4.0)$ & $4.0(2.0-6.0)$ \\
\hline $70-74$ years & $18.5(11.0-27.0)$ & $6.5(6.0-11.0)$ & $3.5(1.5-6.5)$ & $8.0(5.0-10.5)$ \\
\hline$\geq 75$ years & $20.0(14.5-30.5)$ & $8.0(5.0-12.0)$ & $5.0(3.0-8.0)$ & $7.0(4.5-9.5)$ \\
\hline & $P=0.04^{* *}$ & $P=0.006^{* *}$ & $P=0.122^{* *}$ & $P=0.286^{* *}$ \\
\hline
\end{tabular}

Note: *Mann-Whitney U test for PCS differences between genders

**Kruskal-Wallis $\mathrm{H}$ test for PCS differences between age groups

The median for females was $2.5(0.0-5.0)$ and for males 0.0 (0.0-3.0) being statistically significantly higher for females than for males ( $\mathrm{P}=0.024)$. The results of the follow-up pain score assessments showed a statistically significant difference between genders at 2 hours after the procedure, with the median pain score for females being 4.0 (2.0-5.0) and for males $3.0(0.0-4.0)$ $(\mathrm{P}=0.013)$. Figure 2 shows the levels of pain at 2 hours post procedure for different genders. Pain scores on other followup time points did not have a statistically significant difference between genders. Pearson correlation analysis revealed that there were no significant associations between catastrophizing and pain scores during the procedure or at any of the follow-up points, as well as between catastrophizing and patient age. There were also no significant correlations between patients age and pain. However, a Point-Biserial correlation test revealed that there was a positive statistically significant correlation between gender and pain scores during and at 2 hours after the procedure $(r=0.219, \mathrm{P}=0.022$ and $r=0.233, P=0.015$, respectively).

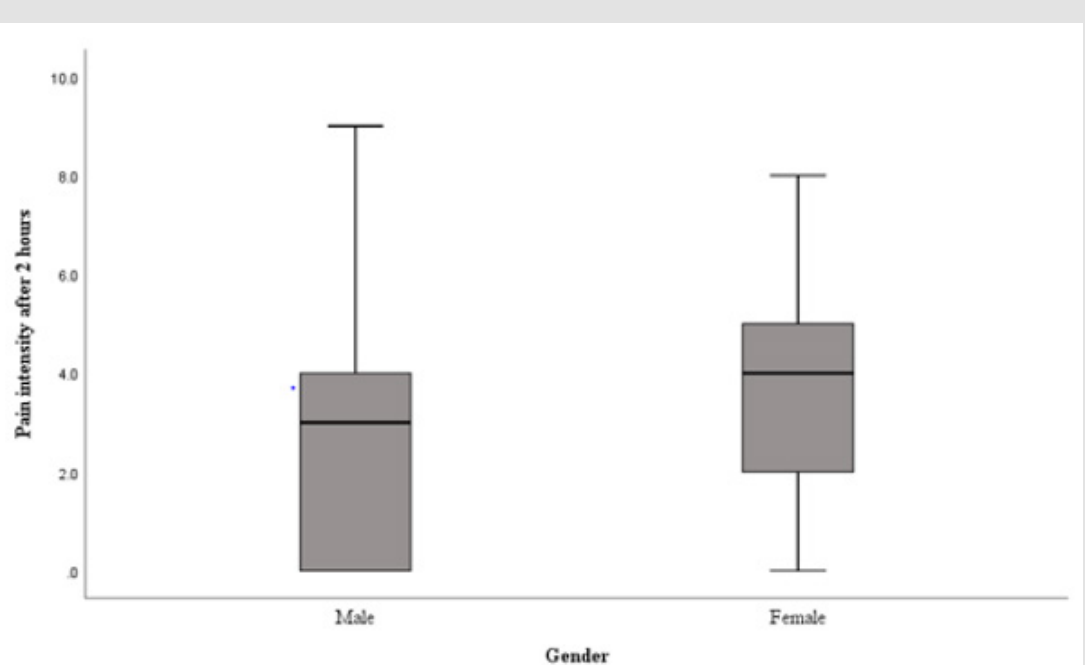

Figure 2: Box plot showing differences in postprocedural pain intensity scores for males and females 2 hours after the intervention.

A linear regression analysis revealed that PCS score had no statistically significant effect on the postprocedural pain score. The results are shown in (Table 4). Univariate regression analysis didn't reveal any statistically significant results regarding higher pain catastrophizing scores and acute access-site pain development. However, our results showed that females had a 3.143 times greater possibility to report access-site pain 1 month after the procedure $(\mathrm{P}$ $=0.038$ ) (Table 5).

Table 4: Linear regression results for PCS predicting acute access-site pain (total PCS score considered as independent variable).

\begin{tabular}{|c|c|c|c|c|c|c|c|c|}
\hline \multirow{2}{*}{$\begin{array}{c}\text { Pain intensity measured on } \\
\text { NRS }\end{array}$} & \multirow{2}{*}{ B } & \multicolumn{2}{|c|}{$95 \% \mathrm{CI}$ for B } & \multirow{2}{*}{$S E B$} & \multirow{2}{*}{$\beta$} & \multirow{2}{*}{ Sig. } & \multirow{2}{*}{$\mathbf{R}^{2}$} & \multirow{2}{*}{$\Delta \mathbf{R}^{2}$} \\
\hline & & LB & UB & & & & & \\
\hline 2 hours after $\mathrm{PCI}$ & 0.032 & -0.01 & 0.074 & 0.021 & 0.146 & 0.129 & 0.021 & 0.012 \\
\hline 12 hours after PCI & 0.004 & -0.032 & 0.04 & 0.018 & 0.021 & 0.827 & 0 & -0.009 \\
\hline 24 hours after PCI & 0.004 & -0.024 & 0.032 & 0.014 & 0.026 & 0.79 & 0.001 & -0.009 \\
\hline 48 hours after PCI & 0.003 & -0.023 & 0.028 & 0.013 & 0.019 & 0.843 & 0 & -0.009 \\
\hline 1 week after PCI & 0.004 & -0.021 & 0.029 & 0.013 & 0.028 & 0.769 & 0.001 & -0.009 \\
\hline 1 month after PCI & -0.009 & -0.032 & 0.014 & 0.012 & -0.074 & 0.443 & 0.006 & -0.004 \\
\hline
\end{tabular}

Note: $\mathrm{B}=$ unstandardized regression coefficient; $\beta=$ standardized coefficient; sig. = significance; $\mathrm{R}^{2}=$ coefficient of determination; $\Delta \mathrm{R}^{2}=$ adjusted $\mathrm{R}^{2}$. 


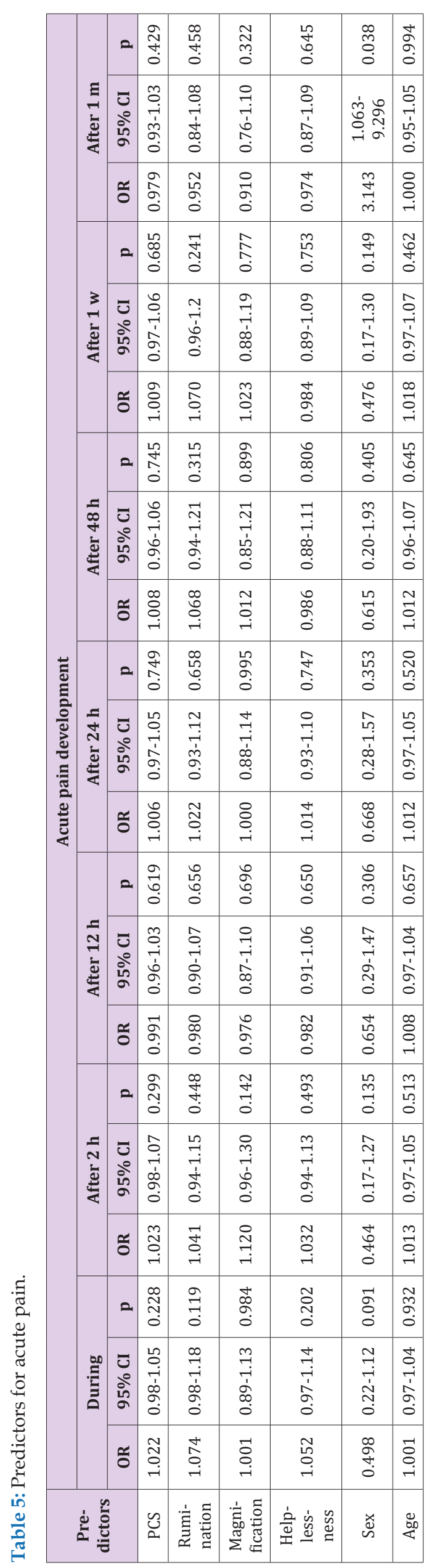




\section{Discussion}

Our study evaluated the gender differences regarding PCS scores and pain scores during and up to 1 month after the intervention. We found that females had a statistically significantly higher PCS score than males, as well as statistically significantly higher pain scores at 2 hours after the procedure. The tests also revealed that females are more likely to have a 1.064-point higher pain score than males 2 hours after the procedure. Differences between genders regarding pain and catastrophizing have been explored in earlier studies, which often reported higher pain and catastrophizing ratings in females 13. According to literature, women may be more sensitive and less tolerant to painful stimuli due to differences in sex hormones and immunity [12-14]. Further study is needed to explore benefit of intervention approach based on gender. Considering age and PCS scores, we found that PCS scores were statistically significantly different between age groups and the highest among those older than 75 years.

Older adults often struggle with medical comorbidities, disability, depression and anxiety and an overall impaired quality of life, and all of these factors could influence catastrophizing thoughts $[15,16]$. Suren et al. didn't find any differences in PCS among different age groups, however Park et al. found that risk for catstrophizing increases with age, although the results were not statistically significant $[16,17]$. It's important to note that in the Suren et al. study the oldest patient was 69 years old, and our study showed highest catastrophizing scores in patients older than 75 years old. Therefore, further research is needed in order to investigate the relationship between age and PCS. Our primary goal of this study was to determine whether the scores of pain catastrophizing were associated with acute pain up to 1 month after PCI and whether the PCS could be used to predict the postprocedural acute pain after the percutaneous coronary intervention. We did not find significant association between PCS score and pain during and after the procedure.

Majority of the studies on postoperative pain are performed with patients after surgeries of great extent and there are limited data on PCS and postprocedural pain [18,19]. While isolated acute pain levels could be comparable between such surgeries and minor procedures, extensity of tissue trauma is considerably larger compared to that experienced by patients after transradial PCI. We came to a conclusion that this is the main reason for insignificant association mentioned above. Many researchers analyze pain catastrophizing and it's association with pain. Some studies show that pain catastrophizing could be associated with chronic pain, delayed recovery and higher disability rates [2022]. Authors suggest that pain catastrophizing might contribute to pain sensitivity through the central nervous system processing of pain-related information, however clear mechanisms are not yet identified 21. Nonetheless, not many studies examine the relationship between pain catastrophizing and acute pain.

Khan et al. performed a study with patients scheduled for an open heart surgery with the aim to establish the relationship between catastrophizing and postoperative pain intensity. Their results indicated that patients showing higher scores on preoperative PCS also tended to report greater postoperative pain intensity [23]. Sommer et al., who examined the association between preoperative catastrophization and acute pain after ear, nose and throat surgery, came to the conclusion, that catastrophizing is a good predictor of postoperative pain [24]. One of the reasons why our results do not demonstrate any significant association between pain catastrophization scores and postprocedural pain could be that the PCI via the radial artery is a less painful procedure than major surgeries, therefore patients reported lower pain scores. In a study, conducted by Cheng et al., patients reported access site pain level with a median rating of $9.0(0.0-28.5)$ on the $100 \mathrm{~mm}$ long visual analogue scale $3 \mathrm{~h}$ after the procedure $[25,26]$, which is in accordance to our results.

Determining which patients could be more prone to catastrophizing could come of use when deciding whether certain interventions would be of clinical value, as it has been shown that pain catastrophizing can be modified in various surgical settings 26. Importance of such interventions can be highlighted by relationship between higher PCS scores and greater need for postoperative opioids, as found by Sacks, et al. [27]. This study has a few limitations. First, patients filled PCS only prior to the procedure, thus data was not available to examine possible correlation between pain and PCS at all time points. This approach could have been important as it is known that PCS tend to vary depending on recovery [28]. Furthermore, no other possible psychological factors, such as anxiety, were evaluated. Some authors found a significant positive correlation between preoperative anxiety and PCS scores. These psychological factors could potentially add to the prediction of acute pain intensity. Further studies should be considered to specify our findings.

\section{Conclusion}

This study found no significant associations between pain catastrophizing and pain intensity after percutaneous coronary intervention. In addition, we found that females are more likely to report higher pain catastrophizing scores and higher pain scores than males and adults aged 75 and over were found to have higher PCS scores. Further study is needed to evaluate the importance of psychological factors regarding acute postprocedural pain. 


\section{References}

1. Ibánez B, James S, Agewall S, Marco Valgimigli, Christoph Varenhorst, et al. (2017) ESC Guidelines for the management of acute myocardial infarction in patients presenting with ST-segment elevation. Revista Española de Cardiología (English Edition) 70(12): 1082

2. Knuuti J, Wijns W, Saraste A, Christian Funck-Brentano, Eva Prescott, et al. (2019) ESC Guidelines for the diagnosis and management of chronic coronary syndromes. European Heart Journal 41(3): 407-477.

3. Collet JP, Thiele H, Barbato E, Maria Dorobantu, Thor Edvardsen, et al. (2020) ESC Guidelines for the management of acute coronary syndromes in patients presenting without persistent ST-segment elevation. European Heart Journal 42(14): 1289-1367.

4. Kolkailah AA, Alreshq RS, Muhammed AM, Zahran ME, Anas El-wegoud M, et al. (2018) Transradial versus transfemoral approach for diagnostic coronary angiography and percutaneous coronary intervention in patients with coronary artery disease. Zahran - 2016 - The Cochrane Library - Wiley Online Library. Cochrane Database of Systematic Reviews 4(4): CD012318.

5. Liu LB, Cedillo MA, Bishay V, Robert A Lookstein, Aaron M Fischman, et al. (2019) Patient Experience and Preference in Transradial versus Transfemoral Access during Transarterial Radioembolization: A Randomized Single-Center Trial. Journal of Vascular and Interventional Radiology 30(3): 414-420.

6. Aktürk E, Kurtoğlu E, Ermiş N (2014) Comparision of pain levels of transradial versus transfemoral coronary catheterization: A prospective and randomized study. Anadolu Kardiyoloji Dergisi 14(2): 140-146.

7. Khan RS, Ahmed K, Blakeway E, Ara Darzi, Thanos Athanasiou, et al. (2011) Catastrophizing: A predictive factor for postoperative pain. American Journal of Surgery 201(1): 122-131.

8. Leung L (2012) Pain catastrophizing: An updated review. Indian Journal of Psychological Medicine 34(3): 204-217.

9. Turner JA, Aaron LA (2001) Pain-Related Catastrophizing: What Is It? Published online 17(1): 65-71.

10. Wheeler CHB, Williams AC de C, Morley SJ (2019) Meta-analysis of the psychometric properties of the Pain Catastrophizing Scale and associations with participant characteristics. Pain 160(9):1946-1953.

11. Sullivan MJ (1995) The Pain Catastrophizing Scale User Manual.

12. Hawker GA, Mian S, Kendzerska T, French M (2011) Measures of adult pain: Visual Analog Scale for Pain (VAS Pain), Numeric Rating Scale for Pain (NRS Pain), McGill Pain Questionnaire (MPQ), Short-Form McGill Pain Questionnaire (SF-MPQ), Chronic Pain Grade Scale (CPGS), Short Form-36 Bodily Pain Scale (SF. Arthritis Care \& Research 63(S11)

13. Papaioannou M, Skapinakis P, Damigos D, Mavreas V, Broumas G, et al. (2009) The Role of Catastrophizing in the Prediction of Postoperative Pain. Pain Medicine 10(8): 1452-1459.

14. Rosen S, Ham B, Mogil JS (2017) Sex differences in neuroimmunity and pain. Journal of Neuroscience Research 95(1-2): 500-508.

ISSN: 2574-1241

DOI: 10.26717/BJSTR.2021.35.005726

Lolita Grygalyte. Biomed J Sci \& Tech Res

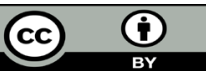

This work is licensed under Creative Commons Attribution 4.0 License

Submission Link: https://biomedres.us/submit-manuscript.php
15. Cederbom S, Leveille SG, Bergland A (2019) Effects of a behavioral medicine intervention on pain, health, and behavior among communitydwelling older adults: a randomized controlled trial. Clinical Interventions in Aging.

16. Park SJ, Lee R, Yoon DM, Yoon KB, Kim K, et al. (2016) Factors associated with increased risk for pain catastrophizing in patients with chronic neck pain. Medicine 95(37): e4698.

17. Suren M, Kaya Z, Gokbakan M, Mehtap G Balta, Serkan Dogru, et al. (2014) The Role of Pain Catastrophizing Score in the Prediction of Venipuncture Pain Severity. Pain Practice 14(3): 245-251.

18. Sorel JC, Veltman ES, Honig A, Poolman RW (2019) The influence of preoperative psychological distress on pain and function after total knee arthroplasty. The Bone \& Joint Journal.

19. Sobol Kwapinska M, Bąbel P, Plotek W, Stelcer B (2016) Psychological correlates of acute postsurgical pain: A systematic review and metaanalysis. European Journal of Pain 20(10).

20. Sewell M, Churilov L, Mooney S, Ma T, Maher P (2018) Chronic pelvic pain-pain catastrophizing, pelvic pain and quality of life. Scandinavian Journal of Pain 18(3)

21. Meints SM, Mawla I, Napadow V, Christina Mc Donnell, Junie Carriere, et al. (2019) The relationship between catastrophizing and altered pain sensitivity in patients with chronic low-back pain. Pain 160(4): 833-843.

22. Neblett R (2017) Pain catastrophizing: An historical perspective. Journal of Applied Biobehavioral Research 22(1).

23. Khan RS, Skapinakis P, Ahmed K, Ara Darzi, Thanos Athanasiou, et al. (2012) The Association Between Preoperative Pain Catastrophizing and Postoperative Pain Intensity in Cardiac Surgery Patients. Pain Medicine13(6): 820-827.

24. Sommer M, Geurts JWJM, Stessel B (2009) Prevalence and Predictors of Postoperative Pain After Ear, Nose, and Throat Surgery. Archives of Otolaryngology Head \& Neck Surgery 135(2): 124-130

25. Cheng KY, Chair SY, Choi KC (2013) Access site complications and puncture site pain following transradial coronary procedures: A correlational study. International Journal of Nursing Studies 50(10): 1304-1313.

26. Gibson E, Sabo MT (2018) Can pain catastrophizing be changed in surgical patients? A scoping review. Canadian Journal of Surgery 61(5): 311-318.

27. Sacks HA, Stepan JG, Wessel LE, Fufa DT (2021) The Relationship Between Pain-Related Psychological Factors and Postoperative Opioid Use After Ambulatory Hand Surgery. Journal of Hand Surgery 44(7): 570-576.

28. Golkari S, Teunis T, Ring D, Vranceanu A (2015) Changes in Depression, Health Anxiety, and Pain Catastrophizing Between Enrollment and 1 Month After a Radius Fracture. Psychosomatics 56(6): 652-657.

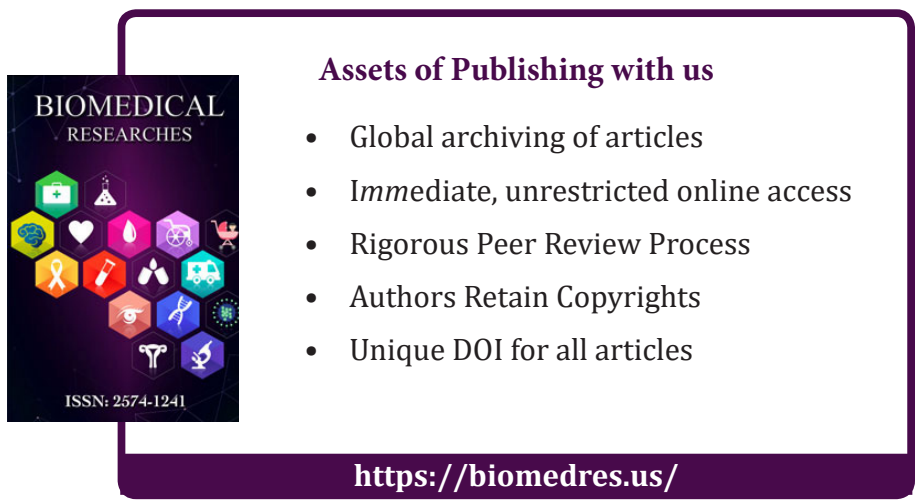

